\title{
Neutrino magnetic moment induced by leptoquarks
}

\author{
Chun-Khiang Chua and W.-Y. P. Hwang \\ Department of Physics, National Taiwan University, Taipei, Taiwan, Republic of China
}

(Received 4 November 1998; published 30 August 1999)

\begin{abstract}
Allowing leptoquarks to interact with both right-handed and left-handed neutrinos (i.e., "nonchiral" leptoquarks), we show that a nonzero neutrino magnetic moment can arise naturally. Although the mass of the nonchiral vector leptoquark that couples to the first generation fermions is constrained severely by universality of the $\pi^{+}$leptonic decays and is found to be greater than $50 \mathrm{TeV}$, the masses of the second and third generation nonchiral vector leptoquarks may evade such constraint and may in general be in the range of $1-100 \mathrm{TeV}$. With reasonable input mass and coupling values, we find that the neutrino magnetic moment due to the second generation leptoquarks is of the order of $10^{-12}-10^{-15} \mu_{\mathrm{B}}$, while that caused by the third generation leptoquarks, being enhanced significantly by the large top quark mass, is in the range of $10^{-10}-10^{-13} \mu_{\mathrm{B}}$. [S0556-2821(99)06509-1]

PACS number(s): 13.10.+q, 12.10.Dm, 14.65.Ha
\end{abstract}

\section{INTRODUCTION}

The existence of a nonzero neutrino magnetic moment has long been a concern of great interest, since it can have an observable laboratory effect such as neutrino-charge lepton elastic scattering, $e^{+} e^{-} \rightarrow \nu \bar{\nu} \gamma$, and also some important astrophysics effects, such as cooling of SN 1987A, cooling of helium stars, etc. It is likely that neutrinos may have a small but nonvanishing mass; for various bounds on magnetic moments and masses, see [1] and [2]. Within the framework of the standard model, a nonzero neutrino mass usually implies a nonzero magnetic moment. It has been shown that [4], for a massive neutrino,

$$
\mu_{\nu}^{\mathrm{SM}}=\frac{3 e G_{F} m_{\nu}}{8 \pi^{2} \sqrt{2}}=3.2 \times 10^{-19} m_{\nu}(\mathrm{eV}) \mu_{B},
$$

where $\mu_{B}$ is the Bohr magneton.

In models beyond the standard model, right-handed neutrinos are often included in interactions (see for a review, e.g., [3]), so that we need not depend on a nonzero neutrino mass to generate a nonzero magnetic moment. In this paper, we consider the possibility of using leptoquark interactions to generate a nonzero neutrino magnetic moment. In many unification models, such as $\mathrm{SU}(5), \mathrm{SO}(10)$, etc., one often puts quarks and leptons into the same multiplet, so that leptoquarks arise naturally for connecting different components within the same multiplet. What makes a leptoquark unique and interesting is that it couples simultaneously to both a lepton and a quark. This may help generate a nonzero neutrino magnetic interaction. Specifically, when a top quark involves in the loop diagram, its mass provides a large enhancement for the neutrino magnetic moment. (In such a diagram, a massless neutrino needs some massive internal fermion to flip its chirality, giving rise to some nonzero magnetic moment.)

We add right-handed neutrinos in the general renormalizable Lagrangian of leptoquarks. Owing to the existence of lepton numbers which recognize the generation, we distinguish leptoquarks by their generation quantum number, but this may induce four-fermion interactions which may en- hance some helicity suppressed process such as $\pi^{+} \rightarrow e^{+} \nu$ to the extent that leptonic universality may even be violated. This usually gives a tight constraint on the leptoquark [5]. For nonchiral vector leptoquarks of electromagnetic strength coupling, this corresponds to having a mass heavier than 50 $\mathrm{TeV}$ for the first generation leptoquark. With such a heavy leptoquark, we still find a nonzero neutrino magnetic moment $\mu_{\nu}$ up to $10^{-18} \mu_{B}$. For the second and third generation leptoquarks, their masses are not severely constrained by the above process. Assuming their mass lying somewhere between 1 and $100 \mathrm{TeV}$, we obtain $\mu_{\nu}$ of the order $10^{-12}-10^{-15} \mu_{\mathrm{B}}$ (from the second generation leptoquark) and $10^{-10}-10^{-13} \mu_{\mathrm{B}}$ (from the third generation leptoquark), respectively. Such predictions may already have some observable effects such as those mentioned earlier.

\section{NEUTRINO MAGNETIC MOMENT IN MODELS WITH LEPTOQUARKS}

Leptoquarks arise naturally in many unification models which attempt to put quarks and leptons in the same multiplet. There are scalar and vector leptoquarks which may couple to left- and right-handed neutrinos at the same time, but only vector leptoquarks can couple to the upper component of the quark $\mathrm{SU}(2)$ doublet. The heaviness of the top quark may enhance the neutrino magnetic moment once we use the vector leptoquark to connect to the quark doublet. Of course, there are subtleties regarding renormalization of the vector leptoquark which may be treated in a way similar to gauge bosons. In our calculation, we adopt Feynman rules in the $R_{\xi}$ gauge and take $\xi \rightarrow \infty$ at the end of the calculation while neglecting all unphysical particles in the $R_{\xi}$ gauge. (This is a step which has often been employed in nonAbelian gauge theories.)

We begin our analysis by constructing a general renormalizable Lagrangian for the quark-lepton-leptoquark coupling. Following [6], we demand such an action to be a $\mathrm{SU}(3) \times \mathrm{SU}(2) \times \mathrm{U}(1)$ invariant which conserves the baryon and lepton numbers but, in addition to [6] we add terms which couple to right-handed neutrinos. For leptoquarks with the fermion number $F \equiv 3 B+L=0$, 


$$
\begin{aligned}
\mathcal{L}_{F=0}= & \left(g_{1 L} \bar{Q}_{L} \gamma^{\mu} L_{L}+g_{1 R} \bar{D}_{R} \gamma^{\mu} l_{R}+g_{1 \nu}^{u} \bar{U}_{R} \gamma^{\mu} \nu_{R}\right) V_{1 \mu}^{(2 / 3)}+\left(g_{2 L}^{d} \bar{D}_{R} L_{L}^{i} i \tau_{2 i j}+g_{2 \nu} \bar{Q}_{L j} \nu_{R}\right) S_{2}^{j(1 / 6)}+\left(g_{2 L}^{u} \bar{U}_{R} L_{L}^{i} i \tau_{2 i j}\right. \\
& \left.+g_{2 R} \bar{Q}_{L j} l_{R}\right) S_{2}^{j(7 / 6)}+g_{3 L} \bar{Q}_{L} \vec{\tau} \gamma^{\mu} L_{L} \vec{V}_{3 \mu}^{(2 / 3)}+g_{1 R}^{u} \bar{U}_{R} \gamma^{\mu} l_{R} V_{1 \mu}^{(5 / 3)}+g_{1 \nu}^{d} \bar{D}_{R} \gamma^{\mu} \nu_{R} V_{1 \mu}^{(-1 / 3)}+\text { c. c. },
\end{aligned}
$$

and, for $F= \pm 2$,

$$
\begin{aligned}
\mathcal{L}_{F=2}= & \left(h_{2 L} \bar{u}_{R}^{c} \gamma^{\mu} L_{L}^{i} i \tau_{2 i j}+h_{2 \nu} \bar{Q}_{L}^{c} i \tau_{2 i j} \gamma^{\mu} \nu_{R}\right) V_{2 \mu}^{(-1 / 6)}+\left(h_{1 L} \bar{Q}_{L}^{i c} i \tau_{2 i j} L_{L}^{j}+h_{1 R} \bar{U}_{R}^{c} l_{R}+h_{1 \nu} \bar{D}_{R}^{c} \nu_{R}\right) S_{1}^{(1 / 3)}+\left(h_{2 L} \bar{D}_{R}^{c} \gamma^{\mu} L_{L}^{i} i \tau_{2 i j}\right. \\
& \left.+h_{2 R} \bar{Q}_{L}^{i c} i \tau_{2 i j} \gamma^{\mu} l_{R}\right) V_{2 \mu}^{j(5 / 6)}+h_{3 L} \bar{Q}_{L}^{c i} \vec{i} i \tau_{2 i j} L_{L} S_{3}^{(1 / 3)}+h_{1 R} \bar{D}_{R}^{c} l_{R} S_{1}^{(4 / 3)}+h_{1 \nu} \bar{U}_{R}^{c} \nu_{R} S_{1}^{(-2 / 3)}+\text { c. c. }
\end{aligned}
$$

The notation adopted above is self-explanatory; for example, $S, V$ denote scalar and vector leptoquarks, respectively, the superscript is its average electric charge or the hypercharge $Y$, and the subscript of a leptoquark denotes which $\mathrm{SU}(2)$ multiplet it is in, and the generation index is suppressed. From here it is clear that among those leptoquarks that couple to neutrinos of both chiralities, a radiative $\nu \nu \gamma$ diagram with the exchange of a virtual $U$-type quark can proceed only when accompanied by a vector leptoquark, namely $V_{1 \mu}^{(2 / 3)}$ in $\mathcal{L}_{F=0}$ or $V_{2 \mu}^{(-1 / 6)}$ in $\mathcal{L}_{F=2}$; on the other hand, the exchange of a virtual $D$-type quark can proceed only with the scalar leptoquark, namely $S_{2}^{(1 / 6)}$ in $\mathcal{L}_{F=0}$ or $S_{1}^{(1 / 3)}$ in $\mathcal{L}_{F=2}$. Note that we do not consider mixing between different leptoquarks due to Higgs interactions, which will introduce additional parameters. The diagram in question is shown explicitly in Fig. 1.

Given these couplings, it is straightforward to calculate induced neutrino magnetic moments via one-loop diagrams. To see that the heavy top quark mass can enhance the prediction, we calculate the $\nu \nu \gamma$ diagram with the exchange of up-type quark and $V_{1 \mu}^{(2 / 3)}$, i.e., the first term in $\mathcal{L}_{F=0}$. As one of the standard methods to treat loop diagrams involving massive vecter particles, we use Feynman rules in the $R_{\xi}$ gauge and take $\xi \rightarrow \infty$ at the end of the calculation while neglecting any unphysicical particle. In addition to minimum substitution, we add the term $e Q_{\mathrm{v}} V_{\mu}^{\dagger} V_{\nu} F^{\mu \nu}$ in the Lagrangian, such that the whole $V V \gamma$ coupling is in a form similar to the non-Abelian $W W \gamma$-type coupling, and the procedure results in a finite limit under $\xi \rightarrow \infty$. We obtain, with all couplings chosen to be real,

$$
\mathcal{L}^{\mathrm{eff}}=-\frac{e}{2 m_{e}} \bar{\nu}\left(\frac{\sigma^{\mu \nu}}{2}\right) \nu F_{\mu \nu} F_{2}, \quad\left|\mu_{\nu}\right|=\frac{e}{2 m_{e}} F_{2},
$$
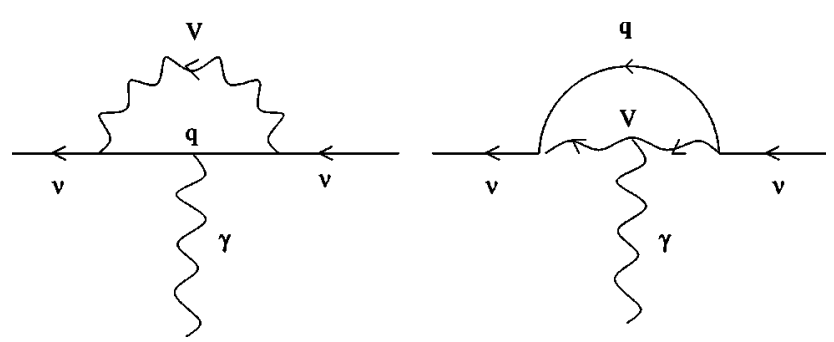

FIG. 1. One-loop diagrams which give rise to a nonzero neutrino magnetic moment.

$$
\begin{aligned}
F_{2}= & \frac{N_{c}}{16 \pi^{2}} \frac{2 m_{e}}{M}\left\{\frac { g _ { L } ^ { u } g _ { \nu } m _ { q } } { M _ { \mathrm { v } } } \left\{Q_{q}\left[f_{1}(a)+f_{2}(a)\right]\right.\right. \\
& \left.+Q_{\mathrm{v}}\left[f_{3}(a)+f_{4}(a)\right]\right\} \\
& +\left(g_{L}^{2}+g_{\nu}^{u 2}\right) \frac{m_{\nu}}{M_{\mathrm{v}}}\left\{Q_{q}\left[g_{1}(a)+g_{2}(a)\right]\right. \\
& \left.\left.+Q_{\mathrm{v}}\left[g_{3}(a)+g_{4}(a)\right]\right\}\right\},
\end{aligned}
$$

where $a=m_{q}^{2} / M_{\mathrm{v}}^{2}, Q_{q}=-Q_{\mathrm{v}}=2 / 3, e>0$ and $N_{c}=3$, while $f_{i}$ and $g_{i}$ are given by

$$
\begin{aligned}
& f_{1}(a)=\frac{2\left[-1+a^{2}-2 a \ln (a)\right]}{(a-1)^{3}}, \\
& f_{2}(a)=-\frac{a\left[3-4 a+a^{2}+2 \ln (a)\right]}{2(a-1)^{3}}, \\
& f_{3}(a)=-\frac{3\left[-1+4 a-3 a^{2}+2 a^{2} \ln (a)\right]}{2(a-1)^{3}}, \\
& f_{4}(a)=-1 / 2, \\
& g_{1}(a)=\frac{\left[-4-5 a^{3}+9 a+6 a(2 a-1) \ln (a)\right]}{6(a-1)^{4}}, \\
& g_{2}(a)=\frac{a\left[3-4 a+a^{2}+2 \ln (a)\right]}{4(a-1)^{3}}, \\
& g_{3}(a)=\frac{\left[7-33 a+57 a^{2}-31 a^{3}+6 a^{2}(3 a-1) \ln (a)\right]}{12(a-1)^{4}}, \\
& g_{4}(a)=\frac{\left(2-6 a+15 a^{2}-14 a^{3}+3 a^{4}+6 a^{2} \ln (a)\right]}{12(a-1)^{4}} .
\end{aligned}
$$

Note that one obtains the desirable chiral structure for the magnetic moment interaction in two different ways: the first is to have an odd number of mass insertions of the quark mass term, giving rise to the first term in $F_{2}$; the other way is by the neutrino mass term, resulting in the second term of $F_{2}$. There are two advantages with the first scenario. First of all, one can obtain a nonzero magnetic moment without be- 
ing restricted by the very light neutrino mass. Second, one may have a prediction enhanced considerably by the heavy top quark mass.

It is tempting to generate a neutrino mass radiatively, to do so one must somehow render the divergence of the related Feynman diagrams to a finite number. Therefore, no mass counterterm is needed and there is no neutrino mass term in the Lagrangian, and neutrino mass is no longer a parameter of the Lagrangian but a quantum prediction [7]. For example, one can let three different $V_{1 \mu}^{(2 / 3)}$ couple to three terms, respectively, in the first line of $\mathcal{L}_{F=0}$ in Eq. (2) and introduce mixings between these leptoquarks, where the degree of divergence is reduced by a GIM-like mechanism. Note that the chiral structure of the mass term is similar to that of the magnetic moment term, and the radiative neutrino mass is also enhanced by the large top quark mass. In such a case, one always has a large neutrino mass accompanying a large neutrino magnetic moment. However, in this paper we do not distinguish those $V_{1 \mu}^{(2 / 3)}$ as different leptoquarks nor do we introduce any mixing mechanism, so the graphs that correspond to neutrino mass correction is divergent and one needs to renormalize it. Therefore, we do not have any prediction about the neutrino mass, indeed it can remain light.

\section{CONSTRAINTS AND NUMERICAL RESULTS}

Before working out numerical predictions, we need to consider the constraints arising from the leptonic decays of the pseudoscalar meson, such as $\pi^{+} \rightarrow e^{+} \nu$ [5]. Integrating out $V^{(2 / 3)}$ and performing Fierz reordering, we obtain $\mathcal{L}_{\text {eff }}$ relevant to the leptonic decay of a pseudoscalar meson:

$$
\begin{aligned}
\mathcal{L}_{\text {eff }}= & \frac{1}{M_{\mathrm{V}}^{2}}\left(2 g_{1 L}^{*} g_{1 R} \bar{D}_{R} U_{L} \bar{\nu}_{L} l_{R}+2 g_{1 \nu}^{u *} g_{1 L} \bar{D}_{L} U_{R} \bar{\nu}_{R} l_{L}\right. \\
& \left.-g_{1 L}^{*} g_{1 L} \bar{D}_{L} \gamma^{\mu} U_{L} \bar{\nu}_{L} \gamma_{\mu} l_{L}-g_{1 \nu}^{u *} g_{1 R} \bar{D}_{R} \gamma^{\mu} U_{R} \bar{\nu}_{R} \gamma_{\mu} l_{R}\right) \\
& + \text { c.c. }
\end{aligned}
$$

We consider the universality constraint arising from the $\pi^{+}$ leptonic decay, and neglect the neutrino mass contribution. Define $R=\operatorname{Br}\left(\pi^{+} \rightarrow e^{+} \nu\right) / B r\left(\pi^{+} \rightarrow \mu^{+} \nu\right)$. The first and third terms of $\mathcal{L}_{\text {eff }}$ have interference with the standard model Fermi interaction. This is an order of $1 / M_{\mathrm{v}}^{2}$ correction to $R$, while the other term is a correction of the order $1 / M_{\mathrm{v}}^{4}$. Furthermore, the first term, which is the scalar coupling, is enhanced by a factor of $m_{\pi}^{2} /\left[\left(m_{u}+m_{d}\right) m_{e}\right]$, so this is the dominant term to constrain the mass of the leptoquark. We assume $g_{1 \nu}^{u}=g_{1 L}=g_{1 R}=g$ which is a natural assumption for the vecter leptoquark. We obtain

$$
R^{\exp }=R^{\mathrm{sm}}\left[1+2 \frac{m_{\pi}^{2}}{m_{e}\left(m_{u}+m_{d}\right)}\left(-\frac{g_{1 L}^{*} g_{1 R}}{\sqrt{2} M_{\mathrm{V}}^{2} G_{F}}\right)\right],
$$

where experimental average $R^{\exp }=(1.230 \pm 0.004) \times 10^{-4}$ [1], and standard model calculation $R^{\mathrm{sm}}=(1.2352 \pm 0.0005)$ $\times 10^{-4}[8]$. This corresponds to

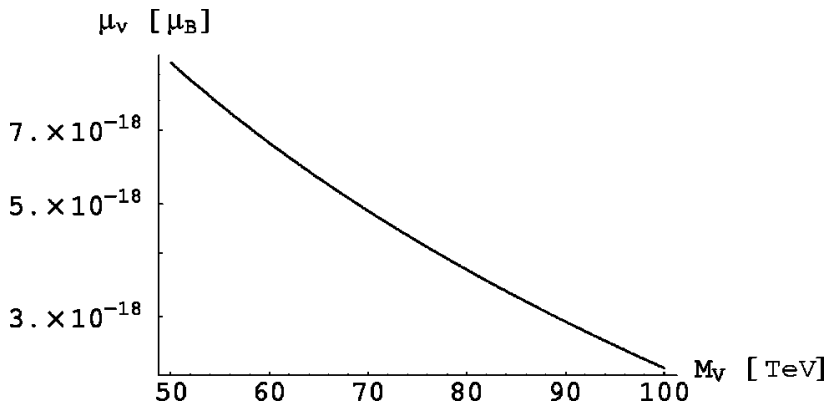

FIG. 2. The first generation neutrino magnetic moment $\mu_{\nu}$ in units of $\mu_{B}$ plotted as a function of the vector leptoquark mass in the range of $50-100 \mathrm{TeV}$.

$$
M_{\mathrm{V}}>g m_{\pi} \sqrt{\frac{\sqrt{2}}{0.0075 G_{F} m_{e}\left(m_{u}+m_{d}\right)}} \sim 50\left(\frac{g}{e}\right) \mathrm{TeV} .
$$

For a coupling of the electromagnetic strength, this corresponds to having the vector leptoquark with a mass greater than $50 \mathrm{TeV}$ for the first generation. This constraint is in fact more severe than what we may obtain from the atomic parity violation experiment, which we shall ignore in this paper. For the second and third generations leptoquarks, there is no direct restriction from the universality of the $\pi$ leptonic decay, nor from the atomic parity violation experiment. Nevertheless, one can find various lower bounds for the leptoquark mass [1], from direct searches at the DESY ep collider HERA, the Fermilab Tevatron $p \bar{p}$ collider, and at the CERN $e^{+} e^{-}$collider LEP. Typical bounds from direct searches are about few hundreds $\mathrm{GeV}$, while the bounds from indirect searches are given in [9]. We shall consider a leptoquark mass in the general range of TeV's.

For the reason of comparisons, let us recall briefly some of the upper limit obtained from the leptonic scattering such as elastic $\nu(\bar{\nu})$ with $l^{+}\left(l^{-}\right), e^{+} e^{-} \rightarrow \nu \bar{\nu} \gamma$, etc., and also from the astrophysical processes such as cooling of helium stars, red giant luminosity, and so on [1]. As a reference point, we recall the standard model formula on the neutrino magnetic moment arising from a nonzero neutrino mass [4], $\mu_{\nu}^{\mathrm{sm}}=3.2 \times 10^{-19} m_{\nu}(\mathrm{eV}) \mu_{B}$ (referred to as "the extended standard electroweak theory"). Accordingly, the upper limit of $\mu_{\nu}$ for the first generation neutrino is $\mu_{\nu}^{\mathrm{sm}} \leqslant 2.3$ $\times 10^{-18} \mu_{B}$ with $m_{\nu} \leqslant 7.3 \mathrm{eV}$. The upper limit may also be obtained from leptonic scatterings, which is typically $10^{-10} \mu_{B}$, or from astrophysics studies with a more stringent upper limit of $10^{-11} \mu_{B}$. Our numerical results for the first generation are summarized in Fig. 2, where the neutrino magnetic moment $\mu_{\nu}$ in units of $\mu_{B}$ is shown as a function of the leptoquark mass. We note that, for the leptoquark mass $\left[V_{1 \mu}^{(2 / 3)}\right]$ of $50-100 \mathrm{TeV}, \mu_{\nu}$ is of the order $10^{-18} \mu_{B}$, a value compatible with the extended standard electroweak theory.

The upper limit of $\mu_{\nu}$ for the second generation neutrino is $0.51 \times 10^{-13} \mu_{B}$ (with $m_{\nu} \leqslant 0.17 \mathrm{MeV}$ ) in the extended standard electroweak theory [1], or in the range of $10^{-10} \mu_{B}$ from leptonic scatterings, while from astrophysics the typical value is $10^{-11} \mu_{B}$. In Fig. 3, we describe our prediction on 


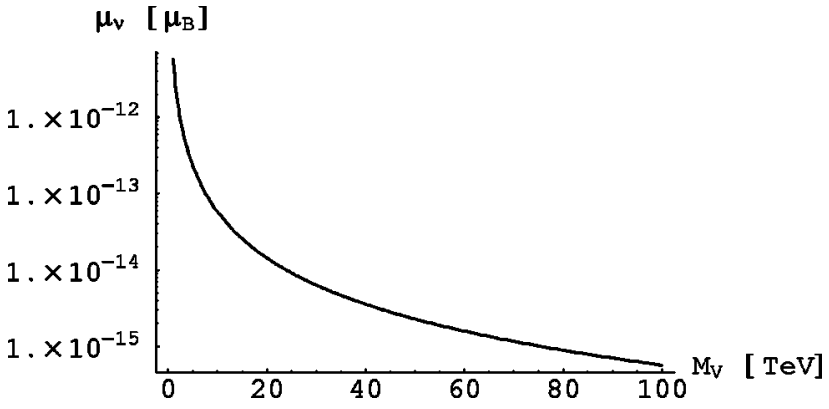

FIG. 3. The second generation neutrino magnetic moment $\mu_{\nu}$ in units of $\mu_{B}$ plotted as a function of the vector leptoquark mass in the range of $1-100 \mathrm{TeV}$.

the neutrino magnetic moment $\mu_{\nu}$ in units of $\mu_{B}$ as a function of the leptoquark mass of $1-100 \mathrm{TeV}$. We obtain $\mu_{\nu}$ around $10^{-12}-10^{-15} \mu_{B}$, a value very close to being observable.

The upper limit of $\mu_{\nu}$ for the third generation neutrino is $1.1 \times 10^{-11} \mu_{B}$ (with $m_{\nu} \leqslant 35 \mathrm{MeV}$ ) in the extended standard electroweak theory [1], or in the range of $10^{-6}-10^{-7} \mu_{B}$ from leptonic scatterings, while from astrophysics studies the upper limit is $10^{-12}-10^{-11} \mu_{B}$. In Fig. 4, we plot the third generation neutrino magnetic moment $\mu_{\nu}$ in units of $\mu_{B}$ as a function of the leptoquark mass in the range of $1-100 \mathrm{TeV}$. We find that $\mu_{\nu}$ is of order $10^{-10}-10^{-13} \mu_{B}$.

\section{CONCLUSION}

Vector leptoquarks in the TeV mass range, when coupled to both left- and right-handed neutrinos, offer an alternative

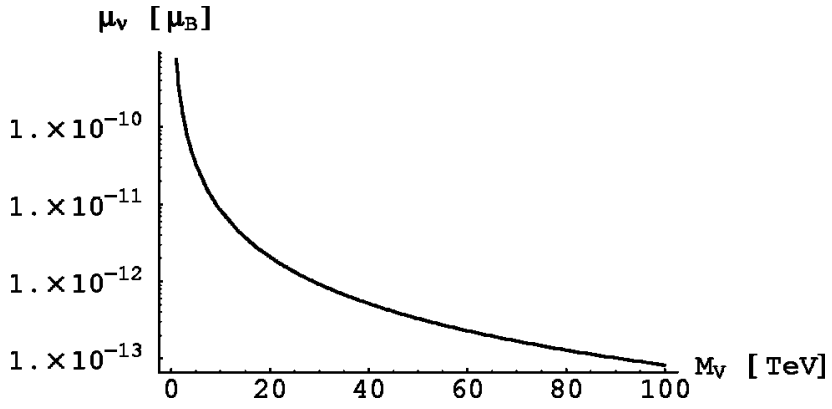

FIG. 4. The third generation neutrino magnetic moment $\mu_{\nu}$ in units of $\mu_{B}$ plotted as a function of the vector leptoquark mass in the range of $1-100 \mathrm{TeV}$.

mechanism for generating a nonvanishing neutrino magnetic moment, which in some cases is by no means negligible. This alternative mechanism (which does not require a nonzero neutrino mass) makes use of the special feature that leptoquarks couple simultaneously to leptons and quarks. For the third generation neutrino, there is a potential enhancement from the very large top quark mass making the corresponding predicted neutrino magnetic moments fairly sizable.

\section{ACKNOWLEDGMENTS}

We would like to acknowledge Dr. C.-T. Chan for valuable discussions. This work was supported in part by a grant from National Science Council of Republic of China (NSC88-2112-M002-001Y).
[1] Particle Data Group, C. Caso et al., Eur. Phys. J. C 3, 1 (1998), and references therein.

[2] Super-Kamiokande Collaboration, Y. Fukuda et al., Phys. Rev. Lett. 81, 1562 (1998); Phys. Lett. B 436, 33 (1998). For a recent review of various hints of neutrino mass, see B. Kayser, hep-ph/9810513.

[3] R. N. Mohapatra and P. B. Pal, in Massive Neutrinos in Physics and Astrophysics (World Scientific, Singapore, 1991).

[4] K. Fujikawa and R. E. Shrock, Phys. Rev. Lett. 45, 963 (1980).

[5] O. Shanker, Nucl. Phys. B204, 375 (1982); W. Buchmüller and D. Wyler, Phys. Lett. 117B, 377 (1986).

[6] W. Buchmüller, R. Rückl, and D. Wyler, Phys. Lett. B 191, 442 (1987).

[7] H. Georgi and S. L. Glashow, Phys. Rev. D 7, 2457 (1973); T. P. Cheng and L.-F. Li, ibid. 17, 2375 (1978).

[8] W. J. Marciano and A. Sirlin, Phys. Rev. Lett. 71, 3629 (1993).

[9] S. Davison, D. Bailey, and B. A. Campbell, Z. Phys. C 61, 613 (1994); M. Leurer, Phys. Rev. D 50, 536 (1994); 49, 333 (1994). 